\title{
Versorgungsforschung bei Psoriasis: Modell Deutschland
}

\author{
Health Services Research in Psoriasis - The German Approach
}

Autoren

Institut
M. A. Radtke, M. Augustin

Institut für Versorgungsforschung in der Dermatologie und bei Pflegeberufen (IVDP), Competenzzentrum Versorgungsforschung in der Dermatologie (CVderm), Universitätsklinikum Hamburg-Eppendorf, Hamburg
Bibliografie

DOI http://dx.doi.org/

$10.1055 / \mathrm{s}-0034-1392856$

Akt Dermatol 2015; 41: 349-356

(c) Georg Thieme Verlag KG

Stuttgart · New York

ISSN 0340-2541

\section{Korrespondenzadresse \\ PD Dr. Marc}

Alexander Radtke

Institut für Versorgungsforschung in der Dermatologie und bei Pflegeberufen (IVDP)

Competenzzentrum

Versorgungsforschung in der Dermatologie (CVderm)

Universitätsklinikum

Hamburg-Eppendorf

Martinistraße 52

20246 Hamburg

m.radtke@uke.de

\section{Zusammenfassung \\ $\nabla$}

Psoriasis vulgaris ist eine häufige, im klinischen Bild wie auch im Schweregrad sehr variable Erkrankung, die für viele Patienten eine große Last darstellt. Das therapeutische Spektrum ist breit und durch eine S3-Leitlinie konsentiert. Im weltweit größten Forschungsprogramm zur Versorgung der Psoriasis durch das Competenzzentrum Versorgungsforschung in der Dermatologie (CVderm) wurde in über 30 Einzelstudien ein dif-

\section{Hintergrund \\ $\nabla$}

Mit einer Prävalenz von 2,5\% gehört die Psoriasis vulgaris (Schuppenflechte) zu den bedeutenden Hauterkrankungen in Deutschland [1]. Nicht zuletzt durch das zunehmende Verständnis dieser Entzündungsreaktion als Fehlregulation des Immunsystems und eine in den letzten 10 Jahren weit entwickelte klinische Forschung konnten die therapeutischen Möglichkeiten entscheidend ergänzt werden. Führten die Patienten mit Psoriasis vor 10 Jahren noch ein Schattendasein, so ist die Erkrankung durch eine umfassende Versorgungsforschung in den letzten Jahren zu einer medizinisch wie sozioökonomisch bedeutenden Modellerkrankung geworden. Die Strukturen, Prozesse und Qualität der Versorgung waren in den letzten 10 Jahren Gegenstand intensiver Forschung [2] ( $\boldsymbol{O}$ Abb. 1). Insgesamt wurden in Deutschland mehr als 30 Einzelprojekte zu diesen Themenstellungen durchgeführt unter Nutzung verschiedenster Erhebungs- und Datenquellen (৫ Tab. 1).

Der Bewertung des Nutzens und der Qualität der medizinischen Versorgung kam hierbei eine zunehmende Bedeutung zu. Um diese Bewertung adäquat durchführen zu können und eine verlässliche Planung der Versorgung zu erwirken, waren valide Daten aus der Versorgungsroutine notwendig. Aus dieser Notwendigkeit entwickelte sich ferenziertes Profil der Versorgungsmerkmale erstellt. Darauf aufbauend wurde ein bundesweites Programm zur Verbesserung der Versorgung begonnen, das die Entwicklung regionaler Psoriasisnetze (PsoNet) sowie die Umsetzung der „Nationalen Versorgungsziele Psoriasis 2010-2015“ beinhaltet. Alle Maßnahmen werden wiederum versorgungswissenschaftlich begleitet.

Der vorliegende Artikel stellt das Vorgehen von der Versorgungsanalyse über die Zieldefinition bis zur Versorgungsgestaltung dar.

erstmals eine Versorgungsforschung zur Psoriasis in Deutschland in einem interdisziplinären Setting, das Erkenntnisse zur Kranken- und Gesundheitsversorgung lieferte und dadurch eine erste Kartierung der Versorgung möglich machte [3], womit die Versorgungsqualität verbessert und deren Effizienz erhöht werden sollte.

Eine zentrale Frage, die für unser Gesundheitssystem nur mit deutschen Daten zu beantworten war, ist in der Häufigkeit und dem Behandlungsbedarf zu finden. In den letzten Jahren wurden durch bundesweite epidemiologische Studien unter Nutzung verschiedener Datenquellen und Patientenkohorten Erkenntnisse gewonnen, die frühere Daten aus anderen Ländern ablösten [4-8]. Dabei können aus den Versorgungsstudien aufgrund der oft hohen Repräsentativität („externe Evidenz“) auch Schlüsse auf die klinische Verteilung von dermatologischen Merkmalen gezogen werden.

\section{Epidemiologie der Psoriasis \\ $\nabla$}

Mit einer Prävalenz von ca. 2,5\% (dies entspricht etwa 1,8 Millionen Betroffenen) zählt die Psoriasis in Deutschland zu den Volkskrankheiten ( $\bullet$ Tab.2). Die für Deutschland publizierten Daten sprechen für eine Punktprävalenz von ca. 2\% [1]. Die Erstmanifestation erfolgt am häufigsten in 


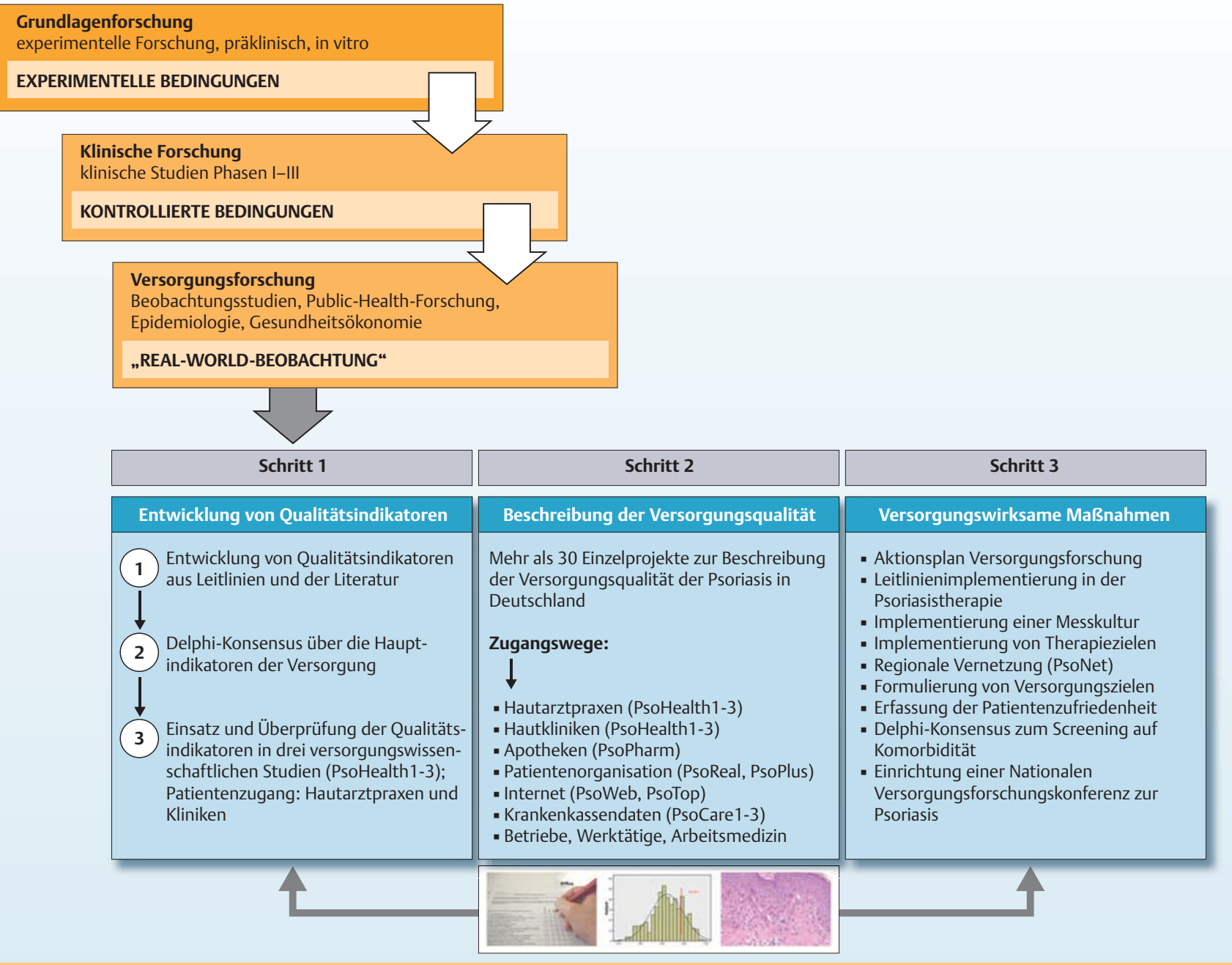

Abb. 1 Gesundheitsforschung am Beispiel der Psoriasis.

der 2. und 3. Lebensdekade, die höchste Prävalenz (>4\%) findet sich im Alter von etwa 40 bis 75 Jahren. Damit sind 50\% der Patienten jünger als 45 Jahre alt, was die sozioökonomische Relevanz in der Zeit der Familienplanung und beruflichen Entwicklung unterstreicht. Mit einer mittleren Lebenserkrankungsdauer von mehr als 40 Jahren stellt die Psoriasis besondere Anforderungen an die Langzeittherapie und an das Krankheitsmanagement. Die Prävalenz bei Kindern liegt nach Sekundärdatenanalysen bei ca. 0,7\% - und zwar fast linear ansteigend von 0,1\% im Kleinkindalter bis 1,4\% im Alter von 18 Jahren [6]. Bereits im Kindesalter ist die Psoriasis somit eine Volkskrankheit, die insbesondere in diesem Alter oft unbeachtet bleibt [9]. Die Psoriasis-Arthritis wurde in drei deutschlandweiten Querschnittstudien bei ca. 20\% der Patienten in dermatologischer Behandlung festgestellt $[4,5]$. Aktuellen Studien zufolge ist die Geschlechtsverteilung nahezu gleich, jedoch zeigt sich bei frühem Krankheitsbeginn eine signifikant häufigere genetische Vorbelastung in Form eines betroffenen Eltern- oder Geschwisterteils. Ist ein Elternteil betroffen, so entwickeln 14\% der Kinder im Laufe ihres Lebens eine Psoriasis im Vergleich zu 3\% bei Kindern ohne elterliche Beteiligung. Sind beide Eltern von einer Psoriasis betroffen, erhöht sich das Risiko für ein Kind auf $41 \%$ bis $75 \%$. Entwickelt ein Geschwisterkind eine Psoriasis, ohne dass die Eltern betroffen sind, so liegt das Risiko, an einer Psoriasis zu erkranken, zwischen 6 und 20\%. Es ist davon auszugehen, dass 30-40\% der Erwachsenen Psoriasispatienten bereits vor dem 16. Lebensjahr Hauveränderungen entwickelt haben.

\section{Versorgungsrelevanz und Krankheitslast}

$\nabla$

Für einen bedeutenden Teil der Patienten geht die Erkrankung oftmals mit schweren Einbußen an Lebensqualität (LQ) einher und stellt für die Betroffenen aufgrund des rezidivierenden und oftmals therapierefraktären Verlaufs sowie der therapieassoziierten Nebenwirkungen eine hohe Belastung dar [10]. Hilflosigkeit, Stigmatisierung, Einschränkungen in der Berufsausübung, Beeinträchtigungen in Freizeit und Alltag, psychische Störungen wie Depression, Angst sowie ein erheblicher negativer Einfluss auf das Sozialleben sind bei etwa einem Drittel der Patienten besonders stark ausgeprägt [11]. Sind exponierte Körperareale betroffen, kann sich der Leidensdruck verstärken [12-15] und der Prozentsatz betroffener Körperoberfläche korreliert nicht mit der Lebensqualität [16]. Der Krankheitsverlauf und die Unzufrieden- 
Tab. 1 Versorgungswissenschaftliche Studien zur Psoriasis durch das CVderm 2004 - 2014

\begin{tabular}{|c|c|c|c|c|c|}
\hline Studie & Thema & $\begin{array}{l}\text { Lebens- } \\
\text { qualität }\end{array}$ & $\begin{array}{l}\text { Psoriasis- } \\
\text { arthritis }\end{array}$ & $\begin{array}{l}\text { Komor- } \\
\text { bidität }\end{array}$ & Kinder \\
\hline PsoAdher & Adhärenz und klinische Outcomes der Biologikatherapie & $x$ & & & \\
\hline PsoAMNOG & Nutzenbewertung von Antipsoriatika & $x$ & $x$ & & \\
\hline PsoArt & Screening auf Psoriasis-Arthritis & & $x$ & & \\
\hline PsoBarrier & Barrieren der Psoriasisversorgung & $x$ & $x$ & $x$ & \\
\hline PsoBest & Das Deutsche Psoriasis-Register $2008-2018$ & $x$ & $x$ & $x$ & \\
\hline PsoCare1 + 2 & Versorgungsstudie an 1,6 Mio. GKV-Versicherten 2003 - 2007 & $x$ & & $x$ & $x$ \\
\hline PsoCare3 + 4 & Versorgungsstudie an 1,5 Mio. GKV-Versicherten 2009 & $x$ & & $x$ & $x$ \\
\hline Psocity & Psoriasis in populationsbezogener Langzeitstudie & $x$ & $x$ & $x$ & \\
\hline PsoCom & randomisierte Studie zum Screening auf Komorbidität & $x$ & $x$ & $x$ & $x$ \\
\hline PsoComp & patienten-relevante Endpunkte bei Psoriasis & $x$ & & & \\
\hline PsoCost & Krankheitskosten und budget impact bei Psoriasis & $x$ & & & \\
\hline PsoDrop & Analyse Arzneimittelpersistenz in der Psoriasisversorgung in Dtschld. & $x$ & $x$ & $x$ & $x$ \\
\hline PsOdyssee & Versorgungsketten und -brüche bei Psoriasis & $x$ & $x$ & & $x$ \\
\hline PsoEpi & Epidemiologie der Psoriasis in Deutschland & $x$ & & & \\
\hline PsoEU & Psoriasisversorgung in Europa & $x$ & $x$ & $x$ & $x$ \\
\hline PsoGoal & randomisierte Studie zu Therapiezielen bei Psoriasis & $x$ & $x$ & $x$ & $x$ \\
\hline PsoGuide & Barrieren der leitliniengerechten Psoriasis-Therapie & $x$ & $x$ & $x$ & \\
\hline PsoHarm & Methoden zum Schadenspotenzial von Antipsoriatika & $x$ & $x$ & & \\
\hline PsoHealth & nationale Studie zur Psoriasisversorgung & $x$ & & & \\
\hline PsoKid & Psoriasisversorgung bei Kindern & & & & $x$ \\
\hline PsoLife & Langzeit-Retentionsraten der Psoriasis & & & & \\
\hline PsoLong & Merkmale der topischen Langzeittherapie & $x$ & & & $x$ \\
\hline PsoMetrics & Outcomes-Messungen und Therapieziele in Behandlung der Psoriasis & & & $x$ & \\
\hline PsoMod & Modellierung der Langzeitverläufe von Psoriasis und -arthritis & $x$ & $x$ & $x$ & \\
\hline PsoNet & Regionale Psoriasis-Netze in Deutschland & $x$ & & & $x$ \\
\hline PsoPharm & Versorgungsstudie $2008 \mathrm{im}$ Apothekennetz & $x$ & $x$ & $x$ & $x$ \\
\hline PsoPlus & Nutzen der Mitgliedschaft in einer Patientenorganisation & $x$ & & & \\
\hline PsoPrefer & Patientenpräferenzen in der Psoriasis-Therapie & $x$ & & & \\
\hline PsoPRO & Methodik der Patienten-Endpunktmessungen & $x$ & $x$ & $x$ & $x$ \\
\hline PsoRapid & Optimierung der Ansprechzeiten von Antipsoriatika & $x$ & $x$ & & \\
\hline PsoReal & Versorgungsstudie 2008 an Selbsthilfepatienten & $x$ & & & \\
\hline PsoSat & Zufriedenheit und Therapieoptimierung bei Psoriasis & $x$ & & & \\
\hline PsoTility & Optimierung von Nutzenmaßen (Utilities) & $x$ & $x$ & $x$ & $x$ \\
\hline PsoTop & Topologie der Psoriasis & $x$ & & $x$ & \\
\hline PsoVac & Impfungen bei Psoriasis & $x$ & $x$ & $x$ & $x$ \\
\hline PsoWeb & Versorgungsstudie im Internet & $x$ & $x$ & $x$ & $x$ \\
\hline PsoWork & Auswirkungen der Psoriasis auf die Produktivität & $x$ & $x$ & & \\
\hline
\end{tabular}

heit mit der Therapie bleiben nicht ohne Auswirkungen auf die Arztwahl. In einer europaweiten Studie gaben ein Drittel der befragten Patienten an, aufgrund ihrer Psoriasis in den letzten zwei Jahren drei oder mehr Ärzte konsultiert zu haben, während 21\% mangels Heilungsaussicht keine Betreuung mehr in Anspruch nahmen [17]. Therapien werden häufig als belastend erlebt, etwa durch topische Therapien, zeitaufwändige UV-Therapien oder unerwünschte Nebenwirkungen systemischer Therapien [18]. Diese Therapielast hat sich als wichtiger Prädiktor des Verlustes an Lebensqualität über die Zeit erwiesen. Hier hat der Erkenntnisgewinn der letzten Jahre deutlich gezeigt, dass durch chronische Leiden nicht wiederherstellbare (irreversible) Schäden und Versäumnisse auftreten können. Dieses im Englischen als „Cumulative Life Course Impairment“ (CLCI) bezeichnete Phänomen führt bei Psoriasis dazu, dass insbesondere junge Patienten verminderte Chancen im Beruf, bei der Partnerwahl und bei der Lebensgestaltung aufweisen [19]. Kommt es zu keinem Ausgleich dieser Defizite (Coping), können sich diese über die Lebenszeit aufaddieren und zu einer kumulierenden lebenslangen Belastung (CLCI) werden. Es wird davon ausgegangen, dass mindestens ein Drittel der von Psoriasis mittelschwer bis schwer betroffenen Patienten entsprechende kumulierende Belastungen irreversibler Art haben.

Im Gegensatz zu vielen anderen chronischen Erkrankungen liegt bei Psoriasis vulgaris das Erstauftreten häufig in der Kindheit und Jugend, und die oft über vier bis sechs Jahrzehnte verlaufende Erkrankung stellt einen kontinuierlichen Belastungsfaktor der Lebensentwicklung dar. Wenngleich dies nicht für alle Patienten im selben Maße gilt und auch Bewältigungsstrategien und persönliche Faktoren gegensteuern können, ist das Ausmaß an bleibender Belastung vor allem dann groß, wenn keine geeignete wirksame Therapie durchgeführt wird.

In der Versorgung gilt es, die bestehenden und sich entwickelnden Belastungen im Sinne des CLCI zu verstehen und Patienten mit erhöhtem Risiko zu erkennen [20].

Psoriasis ist mit erheblichen sozio-ökonomischen Belastungen vergesellschaftet [21]. Die jährlichen Krankheitskosten betragen etwa $9000 €$ bei schweren Formen und 1000 bis $6000 €$ bei leichten bis mittelschweren Formen [22,23] ( $\bullet$ Tab.2). Gründe für die hohen Kosten sind die Aufwendungen für die Arzneimittel sowie 


\begin{tabular}{|c|c|c|}
\hline & Befund & Methodik \\
\hline Prävalenz & deutschlandweit ca. 2 Mio. Menschen & $\begin{array}{l}\text { epidemiologische Analyse } \\
\mathrm{n}=48000\end{array}$ \\
\hline Behandlungsbedarf & $\begin{array}{l}\text { ca. } 90 \% \text { benötigen medizinische } \\
\text { Behandlung }\end{array}$ & $\begin{array}{l}\text { Bedarfsstudien } \\
n=2009 \text { bzw. } 2449\end{array}$ \\
\hline Versorgungsstruktur & $\begin{array}{l}60 \% \text { werden zuerst vom Facharzt } \\
\text { gesehen }\end{array}$ & $\begin{array}{l}\text { Sekundärdaten GKV } \\
\mathrm{n}=1,6 \text { Mio. }\end{array}$ \\
\hline Versorgungszugang & $\begin{array}{l}59,5 \% \text { der schwer Betroffenen } \\
\text { erhalten Systemtherapie }\end{array}$ & $\begin{array}{l}\text { Primärdatenanalyse PsoHealth3 } \\
n=1258\end{array}$ \\
\hline Qualität der Therapie & $\begin{array}{l}\text { nicht leitliniengerecht in 30-50\% } \\
\text { der Fälle }\end{array}$ & $\begin{array}{l}\text { Primärdatenanalysen } \\
\mathrm{n}=2009 \text { bzw. } 2339\end{array}$ \\
\hline Kosten der Therapie & $\begin{array}{l}\text { Kosten/Jahr: ca. 1,5 Mrd. } € \\
\text { Kosten/Patient: ca. } 6600 €\end{array}$ & $\begin{array}{l}\text { Krankheitskostenstudien } \\
n=218206\end{array}$ \\
\hline Patientenzufriedenheit & $\begin{array}{l}\text { ca. } 20 \% \text { der Patienten sind mit der } \\
\text { Versorgung unzufrieden }\end{array}$ & $\begin{array}{l}\text { Versorgungsstudien } \\
\text { zusammengefasst } n=5200\end{array}$ \\
\hline Outcomes & $\begin{array}{l}\text { über } 20 \% \text { der Patienten sehen } \\
\text { Lebensqualität massiv verschlechtert }\end{array}$ & $\begin{array}{l}\text { Versorgungsstudien } \\
\text { zusammengefasst } n=5200\end{array}$ \\
\hline
\end{tabular}

Tab. 2 Fakten zur Psoriasis vulgaris in Deutschland. für stationäre Krankenhausbehandlung, Treiber für indirekte Kosten sind Fehlzeiten am Arbeitsplatz und Produktivitätsausfälle. Durchschnittlich weitere $2000 €$ werden durch Komorbidität ausgelöst. Unter den Morbi-RSA-relevanten Erkrankungen rangiert die Psoriasis auf Rang 63. Der Ausgleichsbetrag für die GKV beträgt für Versicherte mit leichter Psoriasis unter $500 €$, für schwere Formen ca. $7000 €$. Weitere Kostendeterminanten sind die intangiblen Kosten in Form von Lebensqualitätseinbußen und des Leidensdrucks der Patienten [24].

\section{Versorgungsbedarf}

$\nabla$

Versorgungswissenschaftlichen Untersuchungen der letzten Jahre haben gezeigt, dass ein bedeutender Teil der Patienten mit hohem Leidensdruck nicht die verfügbaren und nach Leitlinie vorgesehenen Therapien und Medikamente erhält. Die Gründe hierfür sind vielfältig und Gegenstand der Barriere-Forschung, in der die unterschiedlichen Hürden im Versorgungszugang untersucht werden. Barrieren einer leitliniengerechten Versorgung sind auf Patienten- und Arztseite sowie auf Seiten des Gesundheitssystems zu identifizieren. So ist die mangelnde TherapieAdhärenz eine große Barriere, die v.a. auf mangelnde Aufklärung sowie psychische Faktoren des Patienten zurückzuführen ist [25]. Hindernisse für Ärzte sind vorrangig unvollständige Kenntnis der Leitlinien, eine zunehmende Komplexität bei der Berücksichtigung unterschiedlicher Entscheidungsvariablen in der Therapieplanung sowie ein als unverhältnismäßig empfundener Behandlungsaufwand. Auch ökonomische Limitationen durch teilweise unklare Regelungen in der Kostenerstattung können potenzielle Barrieren darstellen. Regionale und nationale Unterschiede in der Versorgung legen dar, dass diverse, noch nicht beschriebene, Einflussfaktoren innerhalb des Gesundheitssystems existieren [26]. Diesen zufolge tätigen $10 \%$ der Dermatologen in Deutschland nahezu $100 \%$ der Biologika-Verordnungen, deren Einsatz als aufwändig und für die Praxen belastend empfunden wird. Besonders bei Hausärzten und Allgemeinmedizinern kommt hinzu, dass die Leitlinie auf S3-Niveau bislang kaum Verbreitung fand. Dementsprechend verordnete ein großer Anteil der Nicht-Dermatologen am häufigsten systemische Glukokortikosteroide (Kortison) als erste Therapieoption, obwohl diese gar nicht von der Leitlinie empfohlen werden und in den meisten Fällen kontraindiziert sind [27]. Um die Barrieren der leitliniengerechten Versorgung zu überwinden, bedarf es zunächst weiterer systematischer Studien, die sowohl eine neue Komplexität in der Versorgung der Psoriasis untersuchen als auch mögliche Lösungsmodelle diskutieren.

Das CVderm hat innerhalb von zehn Jahren unter Ausnutzung vielfältiger Datenquellen - Arztpraxen, Kliniken, Patientenregistern, Apotheken, Krankenkassen, Patientenorganisationen sowie bevölkerungsbasierten Quer- und Längsschnittstudien - fundamental die Erkenntnisse über die Versorgungssituation in Deutschland erweitert ( $\bullet$ Abb.2). Es konnte gezeigt werden, dass eine Diskrepanz zwischen dem Angebot an effizienten Therapiemöglichkeiten der Psoriasis und der derzeitigen Versorgungssituation besteht. Die Auswahl der Therapieverfahren in der Behandlung der Psoriasis ist oft nicht evidenzbasiert. Es bestehen darüber hinausgehend starke internationale und sogar regionale Unterschiede im Einsatz von Therapieverfahren.

Hinzu kommt der Bedarf nach Früherkennung und Behandlung von Komorbidität. Im Jahr 2005 waren mehr als 80\% der Patienten mit Psoriasis-Arthritis ihre Diagnose nicht bekannt. Die Psoriasis wird immunpathogenetisch zunehmend als Multisystemerkrankung verstanden, analog zu anderen chronisch-entzündlichen Immunerkrankungen wie rheumatoide Arthritis und systemischer Lupus erythematodes. Sie sind mit einem erhöhten Risiko der Entwicklung einer Atherosklerose und weiterer kardiovaskulärer Begleiterkrankungen assoziiert. Auch Stoffwechselerkrankungen, Autoimmunkrankheiten und Depressionen treten bei Personen mit Psoriasis im Vergleich zur Normalbevölkerung signifikant häufiger auf. Dieses erhöhte Komorbiditätsrisiko findet sich bereits bei Kindern. Von besonderer Bedeutung ist unter diesen Erkrankungen bei Psoriasis die Psoriasis-Arthritis, welche in ca. 20\% der Patienten mit Psoriasis der Haut in dermatologischer Behandlung auftritt. Pathogenetische Daten haben Psoriasis als einen eigenständigen Risikofaktor für die Entwicklung einer koronaren Herzerkrankung identifiziert. Epidemiologische Daten aus Deutschland konnten darlegen, dass Patienten mit Psoriasis im Vergleich zur übrigen Bevölkerung 2-3-mal häufiger unter Erkrankungen aus dem Formenkreis des metabolischen Syndroms leiden (Adipositas, Arterielle Hypertonie, Diabetes Typ II, Fettstoffwechselstörung) und signifikant häufiger Assoziationen zu Autoimmunerkrankungen (Arthritis, Morbus Crohn, Colitis ulcerosa) aufweisen [28] ( Tab.3). Je jünger die Patienten sind und je schwerer die Psoriasis ist, desto höher ist das Risiko für kardiovaskuläre Komorbiditäten. Selbst Kinder mit Psoriasis unter 18 Jahren weisen signifikant häufigere Prävalenzen metabolischer Risikofaktoren auf [29]. Im Hinblick auf die Psoriasis bedeutet dies nicht nur die Entzündungsreaktion effek- 


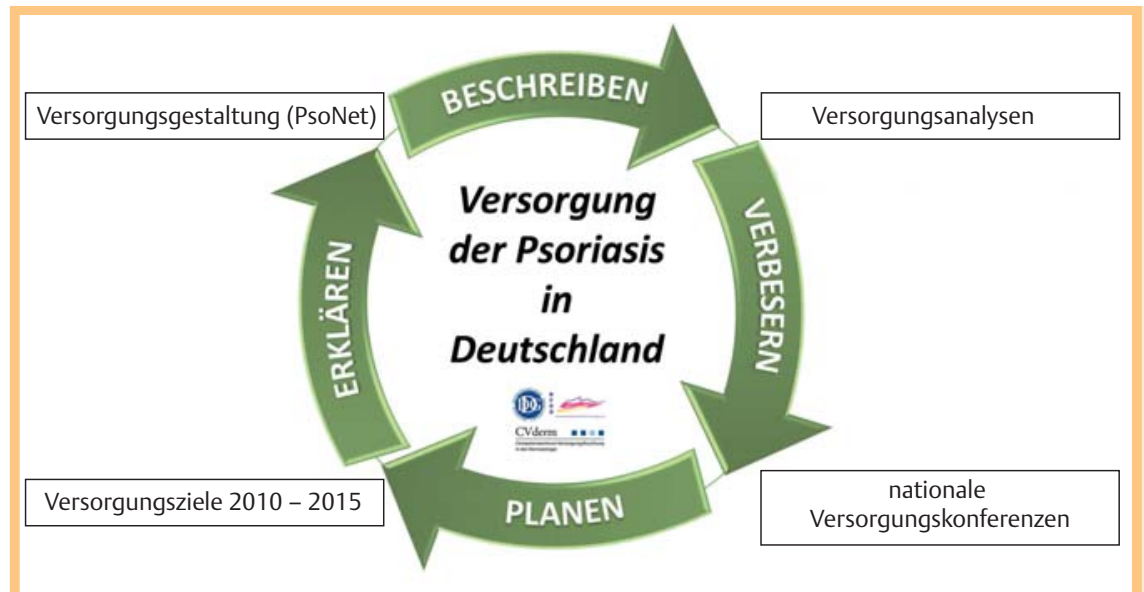

Abb.2 Health Care Research Cycle.

\begin{tabular}{|lccc|}
\hline Erkrankung & $\begin{array}{l}\text { Psoriasis } \\
(\mathbf{n = 3 3 9 8 1 )}\end{array}$ & $\begin{array}{l}\text { ohne Psoriasis } \\
\mathbf{( n = 1 3 1 0 0 9 0 )}\end{array}$ & $\begin{array}{l}\text { Prävalenz-Ratio } \\
\text { (95-\%-Cl) }\end{array}$ \\
\hline Rheumatoide Arthritis & 0,95 & 0,25 & $3,84(3,43-4,31)$ \\
\hline Metabolisches Syndrom & 0,18 & 0,06 & $2,86(2,21-3,71)$ \\
\hline Morbus Crohn & 0,92 & 0,45 & $2,06(1,84-2,31)$ \\
\hline Diabetes mellitus & 12,12 & 6,01 & $2,02(1,96-2,08)$ \\
\hline Colitis ulcerosa & 1,09 & 0,56 & $1,91(1,72-2,11)$ \\
\hline Koronare Herzerkrankung & 13,30 & 7,12 & $1,87(1,82-1,92)$ \\
\hline Hyperlipidämie & 29,88 & 17,05 & $1,75(1,72-1,78)$ \\
\hline Arterielle Hypertonus & 35,61 & 20,57 & $1,73(1,71-1,76)$ \\
\hline Adipositas & 17,82 & 10,39 & $1,72(1,68-1,76)$ \\
\hline Myokardinfarkt & 2,30 & 1,00 & $2,30(2,18-2,42)$ \\
\hline Depression & 16,32 & 8,62 & $1,89(1,78-1,99)$ \\
\hline alle Komorbiditäten (mind. eine) & 57,86 & 34,48 & $1,68(1,66-1,69)$ \\
\hline
\end{tabular}

Tab. 3 Prävalenzraten ausgewählter Komorbidität bei Patienten mit und ohne Psoriasis. tiv zu therapieren, sondern insbesondere auch assoziierte Risikofaktoren zu erkennen und zu beseitigen [30,31].

Die Versorgung der Psoriasis vulgaris wird bei Erwachsenen in Deutschland zu etwa gleichen Teilen von Dermatologen und von Hausärzten (jeweils 40 bis 50\%) geleistet. Ein kleinerer Teil der erwachsenen Patienten wird durch andere Disziplinen, bei Vorliegen von Psoriasis-Arthritis insbesondere durch Rheumatologen, versorgt. Kinder und Jugendliche mit Psoriasis werden zu je etwa einem Drittel durch Dermatologen, Hausärzte und Kinderärzte versorgt $[32,33]$. Sekundärdatenanalysen der BARMER GEK konnten zeigen, dass die Verordnungen für Patienten bei Erstbesuch aufgrund einer Psoriasis vulgaris zu 68\% durch Dermatologen getätigt werden, zu 25\% durch einen Hausarzt und zu lediglich 5\% durch weitere ärztliche Disziplinen. Im Verlaufe der weiteren Quartale kommt es zu einem Rückgang erneuter Besuche, wobei dann der Anteil an Behandlungen durch die Hausärzte relativ steigt und der der Dermatologen fällt. Somit sind Dermatologen und Hausärzte wesentliche Versorger der Psoriasis in Deutschland. Wenngleich aus den Krankenkassendaten keine klinischen Angaben zu Schweregraden und Subtypen der Psoriasis entnommen werden können, spricht die klinische Erfahrung dafür, dass die stationär behandelten Patienten in der Regel besonders schwere Verlaufsformen mit zum Teil schlechtem oder fehlendem Ansprechen auf die Systemtherapie aufweisen. Auch hat sich anhand der erhobenen Querschnittsdaten aus den PsoHealth-Studien gezeigt, dass der Anteil der Krankenhausaufenthalte im Jahr 2013/14 im Vergleich zu 2009 nicht zurückgegangen ist ( Tab.4).
Die Funktion einer umfassenden Versorgung für Patienten mit Psoriasis wird nicht von allen Dermatologen in vertragsärztlicher Praxis gleichermaßen wahrgenommen. Nach den Erkenntnissen des CVderm sind dies etwa 1000 dermatologische Praxen in Deutschland, die hier eine umfassende, leitliniengerechte Vollversorgung leisten. Dabei sind diese Praxen mit allen Belangen der System- und Biologikatherapie vertraut und setzen die modernen Konzepte der Psoriasisversorgung inklusive der Nutzung von diagnostischen und therapeutischen Zielen, objektiver Verlaufsmessung und Management von Nebenwirkungen und Komorbidität voll um [34]. Die Entwicklung der Versorgungsqualität in den letzten Jahren, wie sie durch die PsoHealth-Studien gezeigt wurden, untermauern den Aufwärtstrend nicht nur in der Versorgungsqualität, sondern auch im besonderen Engagement dieser dermatologischen Praxen im Interesse der betroffenen Patienten ( $\bullet$ Tab.3). Die von den Dermatologen selbst gesetzten Versorgungsziele 2010-2015 werden nach den vorliegenden Daten immer besser erreicht - dies in erster Linie auch durch die qualifizierte Versorgung in dermatologischen Praxen.

\section{Therapeutischer Nutzen für den Patienten $\nabla$}

Die Versorgungsforschung hat auch die Erkenntnisse über relevante Nutzen der Therapie geliefert und Patientenpräferenzen identifiziert. Aus 25 standardisierten Nutzen-Items wurden von mindestens $50 \%$ der befragten Patienten die folgenden potenziellen therapeutischen Nutzen am häufigsten genannt [35]: 


\begin{tabular}{|lclll|}
\hline Parameter & $\begin{array}{l}\text { PsoHealth3 } \\
(\mathbf{2 0 1 4 )}\end{array}$ & $\begin{array}{l}\text { PsoHealth2 } \\
\mathbf{( 2 0 0 7 )}\end{array}$ & $\begin{array}{l}\text { PsoReal } \\
\text { (2008) }\end{array}$ & $\begin{array}{l}\text { PsoHealth1 } \\
\text { (2005) }\end{array}$ \\
\hline $\begin{array}{l}\text { mittlerer Schweregrad der Psoriasis (PASI) } \\
\text { mittlere Lebensqualität der Psoriasis (DLQI) }\end{array}$ & 8,1 & 10,1 & $\begin{array}{l}\text { nicht } \\
\text { erfasst }\end{array}$ & 12,0 \\
\hline $\begin{array}{l}\text { Anteil an Patienten mit schwerer Psoriasis } \\
\text { (PASI>20) }\end{array}$ & 9,9 & 7,5 & 6,7 & 8,6 \\
\hline $\begin{array}{l}\text { Anteil an Patienten mit schwerer Einbußen } \\
\text { an Lebensqualität (DLQI> 10) }\end{array}$ & $21,3 \%$ & $28,2 \%$ & $23,6 \%$ & 34,1 \\
\hline $\begin{array}{l}\text { Anteil an Patienten mit vorausgegangener } \\
\text { Systemtherapie }\end{array}$ & $59,5 \%$ & $47,3 \%$ & $49,1 \%$ & 33,0 \\
\hline $\begin{array}{l}\text { Anteil an Patienten mit stationärer } \\
\text { Therapie in den letzten 5 Jahren }\end{array}$ & $20,1 \%$ & $20,1 \%$ & $23,5 \%$ & 23,1 \\
\hline $\begin{array}{l}\text { mittlere Anzahl an Fehltagen am } \\
\text { Arbeitsplatz wegen Psoriasis pro Jahr }\end{array}$ & 3,5 & 4,0 & 8,0 & 3,9 \\
\hline
\end{tabular}

Tab.4 Profil der Versorgungsqualität: Qualitätsindikatoren im Studienvergleich.
- Abheilung aller Hautveränderungen,

- Verminderung von Juckreiz und Brennen an der Haut,

- weniger Zeitaufwand durch die Behandlung,

- Vermeidung starker Nebenwirkungen durch die Behandlung,

weniger Arzt- und Klinikbesuche.

Somit gehen nicht nur von einer wirksamen, sondern auch von einer nebenwirkungs- und belastungsärmeren Therapie besondere Patientennutzen aus [36,37].

Die Auswahl der Therapie und die Definition von Therapiezielen [38] sollten diese individuellen Präferenzen der Patienten berücksichtigen und in eine gemeinsame Therapieplanung Eingang finden [39]. Therapeutische Entscheidungen beruhen in der Medizin traditionell auf objektivierbaren Fakten. Sie werden durch Analyse der persönlichen Merkmale des Patienten sowie aus der externen klinisch-wissenschaftlichen Datenlage gewonnen. Beide Anteile, interne und externe Evidenz, werden in der Evidenz-basierten Medizin als Grundlagen therapeutischer Maßnahmen angesehen. Die Arztperspektive steht dabei im Vordergrund. Eine explizite Rolle für von Patienten berichteten Endpunkten hat diese in den letzten Jahren dominierende Evidenzbasierte Medizin nicht definiert. Dennoch hat die Ergebnismessung aus Patientensicht - international oft unter „Patient reported outcomes" subsummiert - inzwischen weltweit eine immer wichtigere Rolle erhalten. Wesentlich dazu beigetragen hat die Einsicht, dass die Erhaltung oder Verbesserung der Lebensqualität des Patienten eine zentrale Zielsetzung medizinischen Handelns sein sollte. Die Entscheidung des deutschen Gesetzgebers, im GKV-WSG die Bewertung therapeutischen Nutzens als primär patienten-relevanten Nutzen zu formulieren, ist vor diesem Hintergrund zu sehen. Von Ärzten, Entscheidungsträgern im Gesundheitswesen, aber auch Patienten selbst, werden methodische Probleme, Kompetenzprobleme und auch hierarchische Probleme gesehen. So betrachten Kritiker „weiche“ Endpunkte wie Lebensqualität oder Schmerzen als nicht ausreichend quantifizierbar, schon gar nicht objektivierbar, und damit als ungeeignet für die Bewertung therapeutischen Nutzens. Versorgungswissenschaftliche Daten können dies widerlegen.

\section{Maßnahmen}

Unabhängig von jeglicher Unterstützung durch Gesetzgeber, Krankenkassen oder öffentliche Einrichtungen haben die Deutsche Dermatologische Gesellschaft und der Berufsverband der Deutschen Dermatologen eine Handlungsnotwendigkeit erkannt und eine zukunftsweisende Konzeption der verbesserten Versorgung entwickelt. Für die Versorgungsplanung und die zukünftige Allokationsentscheidung war es eine wesentliche Voraussetzung, die Kenntnisse der realen Versorgungsprozesse in der Gesamtbevölkerung sowie in versorgungsrelevanten Gruppen zu analysieren. Vom CVderm und den beteiligten Fachgesellschaften wird angestrebt, die Erkenntnisse der Versorgungsforschung zügig in die Praxis zurückzuführen, beispielsweise durch reibungsarme Implementierung Evidenz-basierter Leitlinien. Ferner wird weiter untersucht, wie den regionalen Unterschieden bei der Versorgung begegnet werden kann. Die versorgungswissenschaftlichen und methodischen Ansätze könnten exemplarisch für andere Fachdisziplinen und insbesondere für andere chronische Erkrankungen sein.

Zur Verbesserung der Versorgungsqualität wurde im Jahr 2009 eine nationale Konferenz zur Versorgung der Psoriasis ins Leben gerufen, die jährlich tagt und „Nationale Versorgungsziele zur Psoriasis 2010-2015“ verabschiedete. Diese lauteten:

1. „Patienten mit Psoriasis haben eine gute Lebensqualität“ 2. „Psoriasis-Arthritis wird frühzeitig erkannt und behandelt“

3. „Komorbidität bei Psoriasis wird frühzeitig erkannt und behandelt"

4. „Psoriasis bei Kindern wird frühzeitig erkannt und behandelt. Sie erlangen eine gute Lebensqualität.“

Diese Ziele stellten eine Selbstverpflichtung der Dermatologen zur Erreichung einer messbar guten Versorgungsqualität dar. Um dies zu gewährleisten, wurden von den deutschen Dermatologen regionale Psoriasisnetze gegründet, von denen bislang 27 aktiv tätig sind (www.psonet.de). Mit Unterstützung der Deutschen Dermatologischen Gesellschaft (DDG) und BVDD sind diese unter dem Namen „PsoNet“ auf regionaler Ebene eingerichtet. Deren Zielsetzung ist die Umsetzung der Nationalen Versorgungsziele Psoriasis 2010-2015 und damit die Verbesserung der Versorgungsqualität auf dem Boden der S3-Leitlinie. Weitere Ziele sind die Verbesserung der Kooperationen zwischen den Arztgruppen und der Kooperation zwischen Kliniken und Praxen. Auch die Früherkennung der Psoriasis-Arthritis sowie der 
assoziierten Komorbidität durch die behandelnden Dermatologen stellt eine Zielsetzung dar, die nur in enger Kooperation mit anderen Disziplinen zu erreichen ist. Den Dermatologen kommt hier eine wichtige Funktion als Weichensteller zu. Die regionalen Psoriasis-Netze unterstützen diese Weichenstellungen durch verbesserte Kooperationen, Bildung von Schnittstellen und interdisziplinäre Behandlungskonzepte. PsoNet steht für eine verbesserte Versorgung durch den leitliniengerechten Einsatz des gesamten Spektrums verfügbarer Therapien. Jeder Dermatologe und jeder kooperierende Kollege anderer Fachrichtungen, der Psoriasis oder dessen Komorbidität behandelt, ist zur Beteiligung an den regionalen Psoriasis-Netzen eingeladen.

Unter maßgeblicher deutscher Beteiligung wurden auf europäischer Ebene die europäischen Versorgungsziele in der Versorgung der Psoriasis und das „European White Paper“ verabschiedet.

\section{Fazit und Ausblick}

\section{$\nabla$}

Im weltweit größten Forschungsprogramm zur Versorgung der Psoriasis konnte durch das Competenzzentrum Versorgungsforschung in der Dermatologie (CVderm) und in Kooperation mit der DDG in über 30 Einzelstudien ein differenziertes Profil der Versorgungsmerkmale erstellt werden. In Anlehnung an dieses Profil wurden Versorgungsziele definiert, die es im kommenden Jahr neu zu konsentieren gilt. Damit diese Versorgungsziele umgesetzt werden können, bedarf es einer Ergebnisbesserung und kontinuierlichen Überprüfung. Die hierfür notwendigen Messverfahren wurden vereinheitlicht und damit bei den Dermatologen eine „Messkultur“ entwickelt. Alle Maßnahmen wurden durch eine systematische Versorgungsforschung mit den notwendigen Fakten unterlegt und im Verlauf durch bundesweite Studien überprüft. Durch diese konzertierten Maßnahmen wurden die Qualität der Versorgung nachhaltig verbessert und die Versorgungsziele zunehmend erreicht. Auf der Basis der zuvor konsentierten Versorgungsindikatoren zeigte sich, dass die Qualität der Versorgung durch Dermatologen zwischen 2005 und 2007 und dann nochmals bis 2009 und 2013 deutlich besser geworden ist. Allerdings bleibt bei mittelschwerer bis schwerer Psoriasis immer noch ein - regional schwankender - Anteil von $20 \%$ bis $50 \%$ ohne leitliniengerechte Versorgung ( Tab.3). Damit weist die Versorgung der Psoriasis zusammengefasst in den letzten Jahren eine erhebliche Verbesserung mit Blick auf die Versorgungsziele auf, wobei noch wichtige Versorgungslücken verbleiben und Anreize für eine leitliniengerechte moderne Versorgung zuweilen fehlen.

Zukunftsaussichten aber auch Verpflichtungen in der Versorgungsforschung sind - neben dem Ausbau der Forschungsinfrastruktur - die weitere gezielte Vernetzung von Forschungseinheiten sowohl auf regionaler als auch nationaler Ebene, um gezielter Daten erheben sowie mögliche regionale Versorgungsunterschiede schneller/besser aufdecken zu können. Neue Datenquellen müssen nutzbar gemacht werden, wobei hier den meist umfangreicheren Sekundärdatenquellen eine besondere Bedeutung zukommt. Dem hohen Bedarf nach qualifizierter Versorgung steht eine immer größer werdende Anzahl an Arzneimitteln zur Verfügung, die sich durch eine hohe Wirksamkeit bei guter Verträglichkeit unter vergleichsweise weniger hohen Kosten auszeichnen.

Deutschland ist damit vorbereitet, um auf den Beschluss der WHO-Generalversammlung reagieren zu können, die am 24. Mai 2014 die Psoriasis weltweit zur versorgungswich- tigen Erkrankung erklärt hat und nun von den Regierungen der Mitgliedstaaten Maßnahmen zur besseren Versorgung fordert.

\section{Was ist zu fordern?}

\section{$\nabla$}

1. Ein allgemein erhöhtes Bewusstsein für die Therapiebedürftigkeit der Psoriasis und ihrer assoziierten Erkrankungen im Kontext einer Systemerkrankung ist zu etablieren, das den Patienten aus der Identität eines trivialisierten „Hautkranken“ wegbewegt und in den Mittelpunkt eines therapeutischen Behandlungsansatzes bringt, der die Risiken und die Behandlungsbedürftigkeit der unterschiedlichen Begleiterkrankungen berücksichtigt.

2. Die generierten Daten werden auch weiterhin Anlass für die Fortführung und weitere Einleitung von Maßnahmen zu einer verbesserten Versorgung sein, die wiederum zu evaluieren ist. In den nächsten Jahren werden die begonnenen Aktivitäten mit Nachdruck fortgeführt, insbesondere der Auf- und Ausbau regionaler Psoriasisnetze und die Umsetzung weiterer nationaler Versorgungsziele. Unabhängig von jeglicher Unterstützung durch Gesetzgeber, Krankenkassen oder öffentliche Einrichtungen haben die Deutsche Dermatologische Gesellschaft und der Berufsverband der Deutschen Dermatologen damit eine zukunftsweisende Konzeption der verbesserten Versorgung bei Psoriasis entwickelt.

3. Forschungsbestrebungen sind zu unterstützen und weiter zu fördern, die zur Identifizierung therapeutischer Zielstrukturen führen, die sowohl simultan die Symptome der Psoriasis effektiv behandeln als auch in systemische Entzündungsreaktionen des Körpers dahingehend modulierend eingreifen, dass auch hier eine Risikominimierung für Folgeerkrankungen erzielt werden kann.

4. Die Grundrechte der Psoriasis-Patienten müssen sich im Versorgungsbedarf reflektiert sehen:

- Gleiche berufliche Möglichkeiten wie für Menschen ohne Psoriasis

- Zugang zur besten Versorgung und adäquater Therapie ohne finanzielle und ortsspezifische Einflüsse

- Zugang zu aktueller, präziser und ausgewogener Information

- Freie Meinungsäußerung zu Behandlungsmethoden

- Anerkennung der Tatsache, dass die sozialen und psychosozialen Belange der Erkrankung ebenso belastend sind wie die physischen Symptome

> Keine soziale Isolation oder Diskriminierung aufgrund der Hautveränderung

\section{Interessenkonflikt}

$\nabla$

M. Radtke und M. Augustin führen oder haben klinische Studien und Projekte zur Versorgungsforschung durchgeführt, die finanziell unterstützt werden oder wurden durch die Firmen JanssenCilag, Pfizer, MSD, Abbvie, Biogen, Medac, Leo Pharma, Almirall, Celgene, Novartis, Amgen.

M. Radtke und M. Augustin sind oder waren als Berater für die Firmen Leo Pharma, Janssen-Cilag, Pfizer, MSD (MSD Sharp \& Dohme), Biogen, Abbott/Abbvie, Almirall, Amgen, Celgene, Novartis, Galderma tätig und haben eingeladene Referententätigkeiten durchgeführt. 
Abstract

\section{Health Services Research in Psoriasis - The German Approach \\ $\nabla$}

Psoriasis is a common disease with a highly variable clinical presentation and disease severity. It is also associated with a significant burden of disease. Therapeutic possibilities are based on the German S3 guideline, which provides evidence-based treatment recommendations. In the worlds largest research program for psoriasis care, conducted by the "German Center for Health Services Research" in Hamburg (CVderm), health care provision has been described by the implementation of more than 25 different studies, conducted nationwide and addressing all aspects of psoriasis care. Based on the results, a program to improve psoriasis care in Germany has been launched under the auspices of the DDG and BVDD. Our action plan called for the establishment of decentralised regional psoriasis networks in order to improve quality of care (PsoNet) and the definition of treatment goals and national objectives in improving health care provision within the next 5 years. The activities are accompanied on a scientific level by the conduction of studies addressing the quality of psoriasis care throughout Germany.

\section{Literatur}

1 Augustin M, Herberger K, Hintzen S et al. Prevalence of Skin Lesions and Need for Treatment in a Cohort of 90880 Workers. Br J Dermatol 2011; 165: $865-873$

2 Augustin $M$, Reich $K$, Reusch $M$ et al. Health services research in psoriasis - the German approach. Dermatology 2009; 218: 293-301

3 Radtke MA, Reich $K$, Blome $C$ et al. Evaluation of quality of care and guideline-compliant treatment in psoriasis. Development of a new system of quality indicators. Dermatology 2009; 219: 54-58

4 Schäfer I, Rustenbach SJ, Zimmer L et al. Prevalence of skin diseases in a cohort of 48,665 employees in Germany. Dermatology 2008; 217: $169-172$

5 Augustin M, Reich K, Glaeske G et al. Comorbidity and age-related prevalence of psoriasis - analysis of health insurance data in Germany. Acta Derm Venereol 2010; 90: 147-151

6 Radtke MA, Reich K, Blome $C$ et al. Prevalence and clinical features of psoriatic arthritis and joint complaints in 2009 patients with psoriasis: results of a German national survey. J Eur Acad Dermatol Venereol 2009; 23: 683-691

7 Reich K, Krüger K, Mössner R et al. Epidemiology and clinical pattern of psoriatic arthritis in Germany: a prospective interdisciplinary epidemiological study of 1511 patients with plaque-type psoriasis. $\mathrm{Br} \mathrm{J}$ Dermatol 2009; 160: 1040-1047

8 Augustin M, Glaeske G, Radtke MA et al. Epidemiology and comorbidity of psoriasis in children. Br J Dermatol 2010; 162: 633-636

9 Radtke MA, Fölster-Holst R, Beikert F et al. Juvenile psoriasis: rewarding endeavours in contemporary dermatology and pediatrics. G Ital Dermatol Venereol 2011; 146: $31-45$

10 Augustin M, Radtke MA. Quality of life in psoriasis patients. Expert Rev Pharmacoecon Outcomes Res 2014; 14: 559-568

11 Augustin M, Krüger K, Radtke MA et al. Disease severity, quality of life and health care in plaque-type psoriasis: a multicenter cross-sectional study in Germany. Dermatology 2008; 216: 366-372

12 Augustin M, Reich $K$, Blome C et al. Nail psoriasis in Germany: epidemiology and burden of disease. Br J Dermatol 2010; 163: 580 - 585

13 Augustin M, Blome C, Costanzo A et al. Nail Assessment in Psoriasis and Psoriatic Arthritis (NAPPA): Development and Validation of a Tool for Assessment of Nail Psoriasis Outcomes. Br J Dermatol 2014; 170: $591-598$

14 Augustin M, Langenbruch AK, Gutknecht M et al. Quality of Life Measures for Dermatology: Definition, Evaluation, and Interpretation. Curr Derm Rep 2012; 1: 148 - 159
15 Radtke MA, Langenbruch AK, Schäfer I et al. Nail psoriasis as a severity indicator: results from the PsoReal study. Patient Relat Outcome Meas 2011; $2: 1-6$

16 Schäfer I, Hacker J, Rustenbach SJ et al. Concordance of the Psoriasis Area and Severity Index (PASI) and patient-reported outcomes in psoriasis treatment. Eur J Dermatol 2010; 20: 62-67

17 Dubertret L, Mrowietz U, Ranki A et al. European patient perspectives on the impact of psoriasis: the EUROPSO patient membership survey. Br J Dermatol 2006; 155: 729-736

18 Langenbruch AK, Radtke MA, Augustin M. Quality of psoriasis care from the patients' perspective - results of the national health care study PsoReal. Eur J Dermatol 2012; 22: 518-524

19 Kimball $A B$, Gieler $U$, Linder $D$ et al. Psoriasis: is the Impairment to a Patient's Life Cumulative? J Eur Acad Dermatol Venereol 2010; 24: 989-1004

20 Augustin M. CLCI: Identifying Patients at Risk. In: Kimball AB, Linder MD, Itin P et al., Hrsg. Dermatological Diseases and Cumulative Life Course Impairment. (Current Problems in Dermatology, Vol. 44). Basel: Karger; 2013: 74-81

21 Augustin M, Radtke MA. Health economic issues in psoriasis. Expert Review of Dermatology 2008; 3: 19-23

22 Berger K, Ehlken B, Kugland B et al. Cost-of-illness in patients with moderate and severe chronic psoriasis vulgaris in Germany. J Dtsch Dermatol Ges 2005; 3: 511-518

23 Sohn S, Schoeffski O, Prinz J et al. Cost of Moderate to Severe Plaque Psoriasis in Germany: A Multicenter Cost-of-illness Study. Dermatology 2006; 212: 137-144

24 Radtke MA, Augustin M. Economic considerations in psoriasis management. Clin Dermatol 2008; 26: 424-431

25 Augustin M, Holland B, Dartsch D et al. Adherence in the treatment of psoriasis: a systematic review. Dermatology 2011; 222: 363-374

26 Radtke MA, Augustin J, Blome C et al. How do regional factors influence psoriasis patient care in Germany? J Dtsch Dermatol Ges 2010; 8: $516-524$

27 Augustin M, Schäfer I, Reich K et al. Systemic treatment with corticosteroids in psoriasis - health care provision far beyond the S3-guidelines. J Dtsch Dermatol Ges 2011; 9: 833-838

28 Augustin $M$, Reich $K$, Glaeske $G$ et al. Co-morbidity and age-related prevalence of psoriasis: Analysis of health insurance data in Germany. Acta Derm Venereol 2010; 90: 147-151

29 Augustin M, Radtke MA, Glaeske G et al. Epidemiology and Comorbidity in Children with Psoriasis and Atopic Eczema. Dermatology 2015; 231 : $35-40$

30 Radtke MA, Mrowietz U, Feuerhahn J et al. Early detection of comorbidity in psoriasis: recommendations of the National Conference on Healthcare in Psoriasis. J Dtsch Dermatol Ges 2015; 13: 674-689

31 Wohlrab J, Fiedler G, Gerdes $S$ et al. Recommendations for detection of individual risk for comorbidities in patients with psoriasis. Arch Dermatol Res 2013; 305: 91 - 98

32 Augustin M, Glaeske G, Schaefer I et al. Versorgungsprozesse der Psoriasis in Deutschland - Langzeitanalyse von Sekundärdaten der gesetzlichen Krankenversicherung [Processes of Psoriasis Health Care in Germany - Long-Term Analysis of Data from the Statutory Health Insurances]. J Dtsch Dermatol Ges 2012; 10: 648-655

33 Augustin M, Reich K, Glaeske G et al. Arzneimittelversorgung von Kindern mit Psoriasis in Deutschland. J Dtsch Dermatol Ges 2013; 11: $751-755$

34 Strömer K, Gass S. Sicherstellung der Versorgung - Psoriasis aus Sicht des Berufsverbandes der Deutschen. gpk Sonderausgabe Gesellschaftspolitische Kommentare 2014; 1: 29-30

35 Augustin M, Radtke MA, Zschocke I et al. The Patient Benefit Index: a Novel Approach in Patient-defined Outcomes Measurement for Skin Diseases. Arch Dermatol Res 2009; 301: 561-571

36 Blome C, Simianer S, Purwins S et al. Time needed for treatment is the major predictor of quality of life in psoriasis. Dermatology 2010; 221: 154-1549

37 Feuerhahn J, Blome C, Radtke MA et al. Validation of the Patient Benefit Index for the Assessment of Patient-Relevant Benefit in the Treatment of Psoriasis. Arch Dermatol Res 2012; 304: 433-441

38 Radtke MA, Reich K, Spehr C et al. Treatment Goals in Psoriasis Routine Care. Archives of Dermatological Research 2015; 307: 445-449

39 Radtke MA, Schäfer I, Blome C et al. Patient benefit index (PBI) in the treatment of psoriasis - results of the National Care Study "PsoHealth". Eur J Dermatol 2013; 23: 212 -217 